\title{
AC 2012-3977: APPLICATIONS OF ARDUINO MICROCONTROLLER IN STUDENT PROJECTS IN A COMMUNITY COLLEGE
}

George Tremberger Jr., Queensborough Community College, CUNY

Raul Armendariz Ph.D., Queensborough Community College, CUNY

Dr. Helio Takai, Brookhaven National Laboratory

Helio Takai is an Elementary Particle and Nuclear Physicist at Brookhaven National Laboratory and an Adjunct Professor at Stony Brook University.

\section{Prof. Todd Holden, Queensborough Community College, CUNY}

Todd Holden is an Associate Professor in the Physics Department of Queensborough Community College of CUNY. His current research interests include bioinformatics and microbial fuel cells. He also mentors student research projects.

Prof. Shermane Austin, Medgar Evers College, CUNY

Dr. Leon P. Johnson, Medgar Evers College, CUNY

Dr. Paul J. Marchese, Queensborough Community College, CUNY

Dr. David H. Lieberman, Queensborough Community College, CUNY

Prof. Tak Cheung, Queensborough Community College, CUNY

Tak Cheung, Ph.D., professor of physics, teaches in Queensborough Community College, CUNY. He also conducts research and mentors student research projects. 


\title{
Applications of Arduino microcontroller in student projects in a community college
}

\begin{abstract}
The Arduino microcontroller has been incorporated in our community college student projects, including interface with GPS receiver for the application of measuring ionospheric disturbance. Our Arduino microcontroller was designed with an ATmega328 chip that enables the training of our community college students to work on the CUNY miniaturized satellite (CubeSat) project at the NASA critical review phase. The Arduino teaching protocol was implemented with our experience in teaching the National Instruments LabVIEW controlled data acquisition board and Lego Mindstorms NXT programming lessons. Particular satellite engineering criteria, such as power consumption, interface algorithm, etc., are highlighted in contrast to those criteria in a typical data acquisition environment. The operation timing issue in CubeSat module necessitates the inclusion of number crunching in the Arduino lessons as well. Arduino microcontroller application was found to be supportive in a laser lab class where students learn about general lab control and data acquisition techniques in addition to their laser work. Less critical applications such as FM detection with Arduino microcontrollers are popular in hobby electronics and readily attract the attention of students in introductory lab sessions. Currently an application of the Arduino microcontroller in field operation is being developed with Brookhaven National Lab to strengthen our engineering technology curriculum. Extension to the use of Arduino microcontroller in atmospheric disturbance studies is also discussed.
\end{abstract}

\section{Introduction}

Arduino microcontroller has been incorporated in our community college student projects including interface with GPS receiver for the application of measuring ionospheric disturbance. The CUNYSAT Microsatellite Project follows the CubeSat protocol ${ }^{1}$, and started about two years ago in collaboration with Cornell University. Electromagnetic wave such as radio-wave that transmits through the ionosphere would have resulted in scattering due to interaction in the ionsphere. The ionospheric influence includes signal speed decrease (ionspheric delays); signal intensity attenuation (fading) and signal phase changes. The degree of influence depends on the density of the electrons that are in the path of the signal, i.e. the total electron content (TEC). Rapid changes in TEC would create rapid GPS signal amplitude fading and phase variations indicating ionospheric scintillations. These signals are more significant in equatorial and polar regions and also have seasonal and time of day variability. Ionospheric scintillations are also a function of solar activity as well as other atmospheric events.

Our Arduino microcontroller was designed with an ATmega328 chip which enables the training of our community college students to work on the CUNY miniaturized satellite at the NASA critical review phase. The Arduino Duemilanove board would serve as a low budget learning tool and provide identical environment for the CubeSat second microprocessor ATmega 328p that controls the GPS instrumentation and 
communications with the master microprocessor MSP430F1612 in Command and Data Handling (CDH). An alternative newer design that combines microprocessors does not change the fact that Arduino provides a solid training platform for our community college students.

\section{Teaching: graphical programming versus text programming}

The Arduino teaching protocol was implemented with our experience in teaching National Instruments LabVIEW controlled data acquisition board and Lego Mindstorms NXT programming lessons. Text programming teaching has been losing ground to graphical programming teaching especially for community college pre-engineering and technology students that needs English skill remediation. One can attribute such trend as the raising popular with iPad, Android, etc. with the new incoming students that generally would have lower scores as compared to the previous incoming class. This paper discusses NXT programming in more details since LabVIEW lessons have been reported by us previously ${ }^{2}$.

Lego provides a toy system (Mindstorms) that allows an instructor to teach the basic principles of robotic movement. Physics provides the basic principles of how things work and optics (microscope, laser) has been opening doors for biology as well as medicine applications. Therefore we have selected those Lego Mindstorms exercises that emphasize acceleration and light detection. Lego Mindstorms uses graphical programming to control the movements and light (vision) data collection. Students having trouble with NXT programming would generally not be able to progress to LabVIEW programming. NASA uses graphical programming (LabView) for control and data collection in critical missions. A special exercise has been implemented to mimic the NASA Mars Pathfinder operating principles. The exercise aims to teach the principles of robotic vision and data communication. The goal is to illustrate the graphical programming control of vision via reflectivity measurement and data communication to third party computing environment such as Excel.

Lego NXT block programming is used to measure reflectivity changes as a function of elapsed time. Vision requires reflectivity measurements. The program reads light intensity reflectivity and elapsed time onto a text file. There are 8 elements in the program as illustrated in Figure 1.

1) The loop element

2) The light sensor element

3) The clock element

4) The number to text conversion element for the clock signal

5) The number to text conversion element for the light signal

6) The text manipulation element for bringing (concatenate) texts together

7) The write data element

8) The close file element 


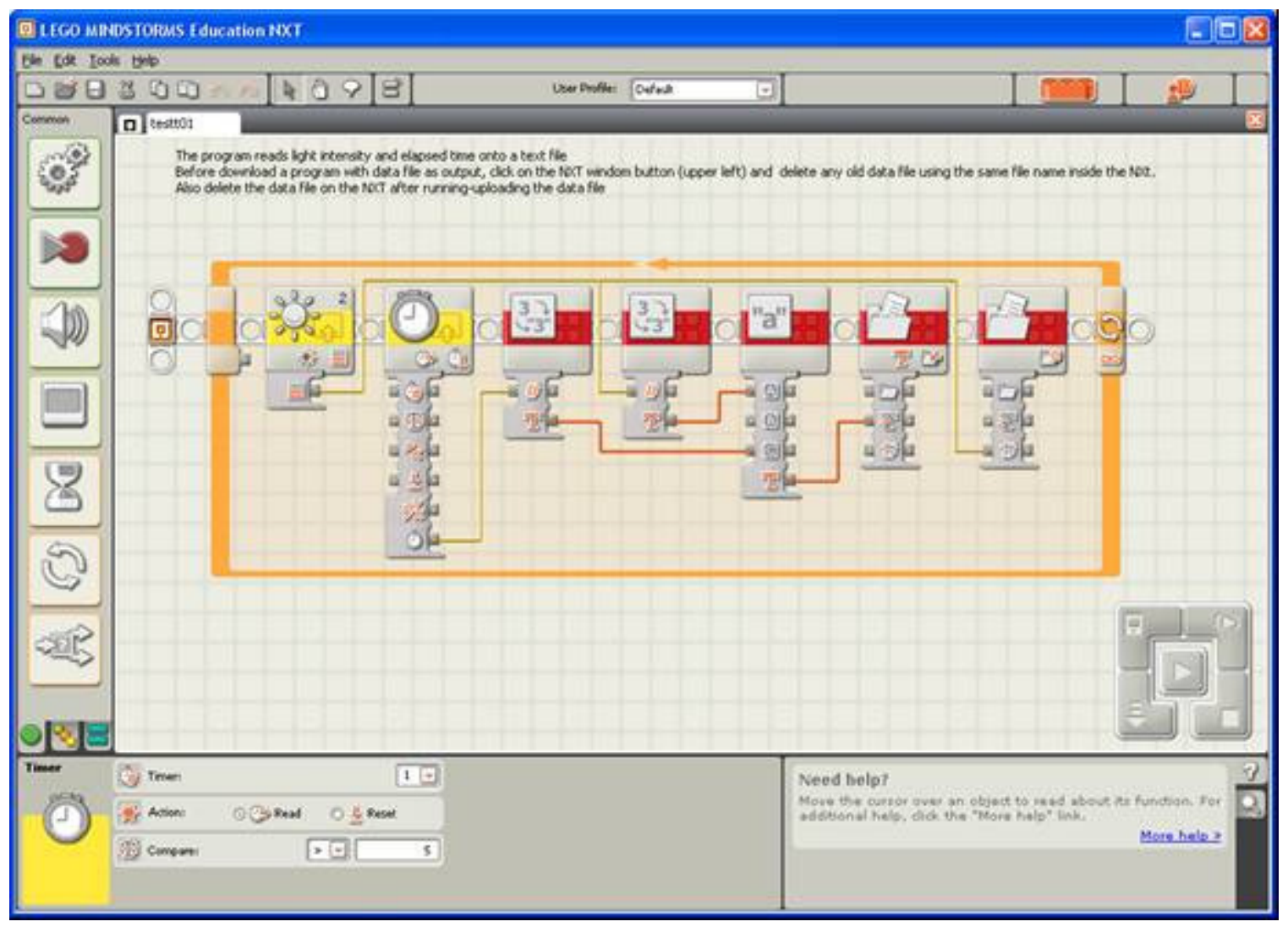

Figure 1: The schematics of a lego block program. The three sentences are: The program reads light intensity and elapsed time onto a text file. Before download a program with data file as output, click on the NXT window button (upper left) and delete any old data file using the same file name inside the NXT. Also delete the data file on the NXT after running-uploading the data file.

All the elements are available on the NXT tool menu. After dragging an element, place the mouse icon at the element bottom such that the mouse icon would change to a doublearrow icon. Drag on the double-arrow icon so that all the available terminals are visible. This step is important for a beginner because then all the wirings would be clearly visible. The property of each element can be easily examined by a click. On the NXT program screen, there is a cluster of controls at the bottom right corner. The center button is for downloading the program onto the NXT. The upper left button is use to examine the connection of the NXT; the stop button is at the left bottom corner. A crucial step has been emphasized, before downloading a program with data file as output, one must click on the NXT window button (upper left) and delete any old data file using the same file name inside the NXT. This step can be eliminated when a user acquires the habit of deleting the data file on the NXT after running-uploading that data file onto a PC. The text data can be exported to Excel for analysis.

After the mastering of NXT programming, the teaching of LabVIEW would follow ${ }^{2}$. The interface of Arduino Duemilanove with LabVIEW was performed using the 
instructions available in open literature ${ }^{3}$. A typical voltage reading on the Arduino Duemilanove output pins in our lab setting is displayed in Figure 2.

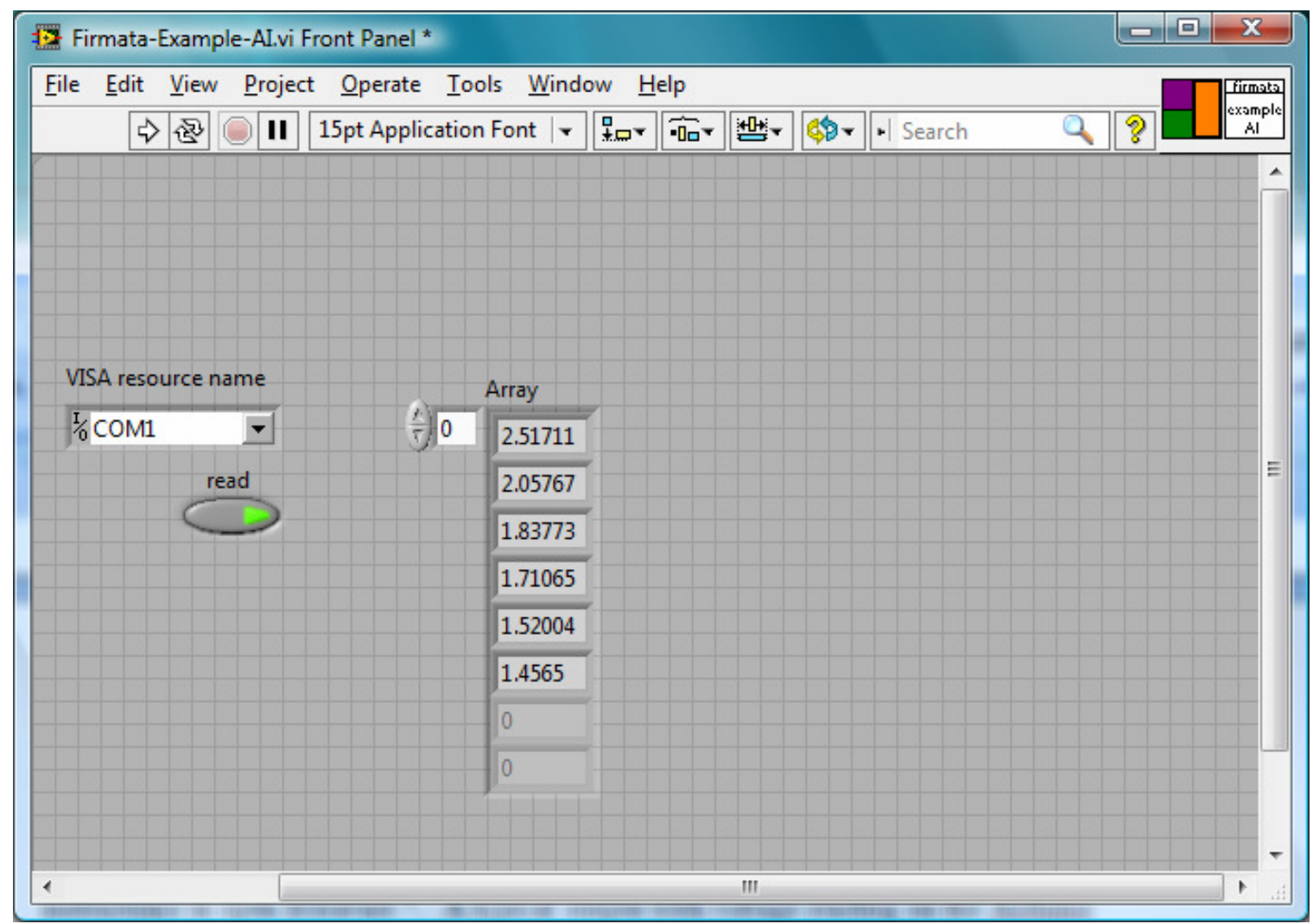

Figure 2: A typical voltage reading in our lab using the Firmata-Example-AI available in open literature $^{3}$. The graphical program wiring is available in Reference 3 as well.

Particular satellite engineering criteria such as power consumption, interface algorithm, etc are highlighted in contrast to those criteria in regular data acquisition environment. The timing issue in CubeSat environment necessitates the inclusion of number crunching in the Arduino lessons or other microprocessors. Teaching experience shows that these demands are usually easier to implement in text programming reserved for advanced students with better working memory capacity ${ }^{4}$. An illustrative example is discussed as the following. A normalized standard deviation is used customarily to represent the GPS signal S4 index.

$$
S 4=\sqrt{\frac{\sum_{x}(\bar{x}-x)^{2}}{N \cdot \bar{x}^{2}}}
$$

where $\bar{x}$ denotes the mean, $\mathrm{x}$ is the current CNR (Carrier/Noise) value, and $\mathrm{N}$ is the total number of samples or the sample window. To simplify the implementation, it would be

$$
s \text {-diff }[+=\operatorname{avg} * \text { avg }-2 * C N R t e m p * a v g+C N R t e m p * C N R t e m p ;
$$


cumsum []$+=$ CNRtemp;

where ' $\mathrm{x}+=\mathrm{y}$ ' means ' $\mathrm{x}=\mathrm{x}+\mathrm{y}$ '. The cumsum [] is a cumulative sum of the CNR values, divide it by the number of samples to calculate a mean. $S$-diff[] is a summation of $(\bar{x}-x)^{2}$. Then S4 is simply:

$$
\begin{aligned}
& \operatorname{avg}=\text { cumsum }[] / N ; \\
& S 4[]=\operatorname{sqrt}(s-\operatorname{diff}[] /(N * \operatorname{avg} * \operatorname{avg})) ;
\end{aligned}
$$

$\mathrm{N}$ could be 20 or more for sufficient averaging. The previous Cornell design recommends two strategies to calculate the S4 index, every 30 seconds, or every 4 minutes. The CUNY CubeSat would be able to track ionospheric irregularity zonal drifts in mid latitude to supplement the study of such drifts at low latitude by Muella, Kintner and others ${ }^{5}$. A 4-minute window is sufficient to track-lock a satellite before recalculating the $\mathrm{S} 4$ for all satellites in view. The 30 -second mode is for the case when a particular satellite falls below the horizon. Anyway such critical numerical steps can be a quite straight forward learning from Arduino text-programming tutorials available on the Arduino web site ${ }^{6}$, although we do teach LAbVIEW text command environment ${ }^{2}$. Experience shows that critical engineering criteria are challenging and rewarding, especially for those who are part of the CUNYSAT Microsatellite Project Critical Review Presentation Teams at NASA/Goddard Institute for Space Studies Feb 18, 2011.

Arduino microcontroller application was found to be supportive in a laser lab class where students learn about general lab control and data acquisition techniques in addition to their laser work. Scanning, coincident counting within detection window, etc are useful examples. Less critical applications such as FM detection with Arduino microcontrollers are popular in hobby electronics and usually attract the attention of students readily in an introductory session. A frequency counter tutorial has been published as well ${ }^{7}$. An application of Arduino microcontroller in field operation is currently being developed with Brookhaven National Lab to strengthen our engineering technology curriculum. Ultrahigh Energy Cosmic Ray detection using RF technology has been an important tool in cosmology ${ }^{8}$. Arduino microcontroller would add decision capability to improve the detection scheme. Extension to the use of Arduino microcontroller in atmospheric disturbance studies is also possible since terrestrial gamma ray flash, etc also would carry RF signatures. Such data would supplement those events collected by RHESSI satellite.

\section{Conclusions}

Arduino microcontroller is a low budget versatile device and most suitable for training community college pre-engineering and technology students in their projects. The use of NXT programming as a starting point for LabVIEW programming has proven to be beneficial to our community college students. Critical engineering criteria offer a challenging and rewarding environment for those advanced students familiar with text programming. 


\section{Acknowledgements}

The support of Mr. Alexei Kisselev is gratefully acknowledged. Partial supports from NSF-REU-0754673, NSF-ATM-0851932, NASA-NNX10AE72G, and the American Recovery and Reinvestment Act of 2009 (ARRA) are gratefully acknowledged. Any opinions, findings, and conclusions or recommendations expressed in this material are those of the authors and do not necessarily reflect the views of the above funding sources.

\section{Bibliographic information}

1. S. Austin, W. Harris, L. Johnson "Transformative strategies for growing diversity in science and technology disciplines" ICERI2009 Proceedings , 2nd International Conference of Education, Research and Innovation , page 1751-1759, 2009 http://library.iated.org/view/AUSTIN2009TRA

2. LabVIEW graphical programming in an introductory engineering physics course, David H. Lieberman, C. Neuman, A. Flamholz, G. Tremberger Jr., P. J. Marchese, D.P. Engelberg, \& T.D. Cheung, Proceedings of the 2006 American Society for Engineering Education Annual Conference \& Exposition, 6 pages, published in CD-ROM format by www.asee.org, 2006

3. http://code.google.com/p/labviewduino/ (last accessed Jan 12 2012)

4. Jaeggi, Susanne M., Buschkuehl, Martin, Perrig, Walter J. and Meier, Beat(2010) "The concurrent validity of the N-back task as a working memory measure”, Memory, 18: 4, 394- 412, 2010

5. Muella, M.T.A.H., E.R. de Paula, I.J. Kantor, I.S. Batista, J.H.A. Sobral, M.A. Abdu, P.M. Kintner, K.M. Groves, and P.F. Smorigo, GPS L-band scintillations and ionospheric irregularity zonal drifts inferred at equatorial and low-latitude regions, J. Atmos. Solar-Terr. Phys., 70(10), 1261-1272, 2008.

6. http://www.ladyada.net/learn/arduino/index.html (last accessed Jan 12 2012)

7. Nawrath, Martin "Arduino Frequency Counter Library" Laboratory for Experimental Computer Science at the Academy of Media Arts Cologne Germany http://interface.khm.de/index.php/lab/experiments/arduino-frequency-counter-library/ (last accessed Jan 12 2012)

8. Takai, Helio (2006-04-26). "MARIACHI — Detecting Ultra High Energy Cosmic Rays with radar". American Physical Society, APS April Meeting, April 22-26, 2006.

http://adsabs.harvard.edu/abs/2006APS..APR.P7009T. (last accessed Jan 12 2012) 